Investigaciones Fenomenológicas, n. 8, 2011, 23-43.

e-ISSN: $1885-1088$

\title{
IDEAS PARA UNA PSICOLOGÍA FENOMENOLÓGICA
}

\author{
Urbano Mestre Sidoncha \\ Universidade da Beira Interior, Covilhã, Portugal \\ umestre@ubi.pt
}

\begin{abstract}
Resumen: En este trabajo hablamos de la posibilidad de una psicología fenomenológica, no como oportunidad de refundición del proyecto husserliano, sino como plan de revisión de los fundamentos de una psicología de confección naturalista a partir de un tratamiento estrictamente fenomenológico. Esta revisión crítica, operada por Husserl, dialogará muy de cerca con las tesis que $\mathrm{W}$. Dilthey desenvuelve en 1894, bajo el signo de una psicología descriptiva. Es nuestra intención mostrar, una vez ponderadas las insuficiencias evidenciadas por la perspectiva diltheyniana, la utilidad incuestionable de la psicología fenomenológica como plataforma y vía de acceso indirecto para una auténtica filosofía fenomenológica.
\end{abstract}

Palabras clave: Psicología descriptiva, psicología fenomenológica, fenomenología trascendental, Dilthey, Husserl.

\begin{abstract}
In this paper, we address the possibility of a phenomenological psycho-logy, not having in mind a re-establishment of the husserlian project, but as plan to revise, from a strictly phenomenological approach, the basis of a naturalistic psychology. Such a critical revise, operated by Husserl, will dialogue closely with the thesis developed by W. Dilthey in 1894, under the title of a descriptive psychology. It's our purpose to explain, given the insufficiency shown by Dilthey's own perspective, the unquestionable utility of this phenomenological psychology as groundwork and indirect access way to a genuine phenomenological philosophy.
\end{abstract}

Keywords: Descriptive psychology, phenomenological psychology, transcendental phenomenology, Dilthey, Husserl.

\section{INTRODUCCIÓN}

El tema que nos ocupará en este trabajo es deliberadamente provocador y no deja de ser revelador el hecho de ser así. Efectivamente, hablar de Ideas para una psicología fenomenológica convoca inmediatamente, y por sí solo, el contenido de sentido del epígrafe del bien conocido texto husserliano de 1913, Ideas directrices para una fenomenología pura y una filosofía fenomenológica, donde está vertida la célebre y más perenne caracterización de la fenomenología como filosofía trascendental. La provocación reside ahí: esa apropiación de sentido del texto de 1913, que aquí preconizamos, parece querer apuntar no en el sentido de una filosofía fenomenológica, como explícitamente prescribe Hus- 
serl, sino en otra dirección, en la de una psicología fenomenológica, que pide que se reescriba la historia y se rediseñe el mapa de la escuela fenomenológica tal cual fuera preliminarmente definida por su fundador, como si ésa fuera la marca de agua de un singular destino que, habiendo permanecido en la sombra, se impone ahora revelar. Una tal idea, lo sabemos bien, surge a partir de 1904 (ésa es por lo menos la confesión implícita de Husserl en el § 70 de Die Krisis der europäischen Wissenschaften und die transzendentale Phänomenologie ${ }^{1}$ ) y, de un modo explícito y abierto, a partir de 1907 -fecha de la entrada en escena del instrumento de la reducción fenomenológica- como expresión de una contradicción evidente y como confesión inmediata de la incomprensión de fondo en relación al sentido último de la marcha de la argumentación seguida por el propio Husserl. Pero si esa sospecha se difundió, eso significa que parte de mi objetivo está ya cumplido:

i) el de convocar la atención del lector para la siempre tensa relación dialógica entre fenomenología y psicología como símbolo del proceso de interna maduración generado en el espacio de la auto-comprensión de la fenomenología y, no menos relevante,

ii) para la cuestión candente de una putativa descaracterización temática y metodológica de la fenomenología siempre que está en causa la tentativa de volverla -en un cierto sentido que escudriñaremos más abajo- "productiva".

En esencia, lo que pretendemos es dirigir nuestro abordaje a la siempre ambigua relación de Husserl con la psicología y, por esto mismo, se trata de un abordaje que no podría olvidar uno de sus capítulos más admirables, hecho realidad precisamente en el propósito de Husserl rtde considerar la posibilidad y necesidad de una psicología pura o fenomenológica. Pero esta idea, en su plena concreción, sólo se deja traer a plena luz del día desenvolviéndose sistemáticamente en el horizonte de sentido más amplio de una fenomenología que, en su versión madura, se plantea como filosofía trascendental. Por otro lado, también queremos interrogar el contenido de sentido de esta exigencia de produc-

1 "[...] Konnte die Epoché als eine ausdrückliche methodische Grundforderung nur eine Sache nachkommender Reflexion dessen sein, der schon in einer gewissen Naivität und aus einer historischen Situation her in die Epoché sozusagen hineingezogen worden ist und schon ein Stück dieser neuen 'Innenwelt', gewissermaßen ein Nahfeld aus ihr, mit einem dunkel vorgezeichneten Fernhorizont, sich zu eigen gemacht hatte. So ist er erst vier Jahre nach Abschluss der Logischen Untersuchungen zum ausdrücklichen, und selbst dann noch unvoll-kommenen Selbstbewusstsein ihrer Methode gekommen". Edmund Husserl, Die Krisis der europäischen Wissenschaften und die transzendentale Phänomenologie, Hua VI, Den Haag, Martinus Nijhoff, 1976, p. 246. Cursivas mías. 
tividad que incesantemente es lanzada sobre la fenomenología y que, a mi entender, ha sido la fuente de equívocos y de bastas interpretaciones.

Aludiendo ahora a este último objetivo, digamos que la necesidad de enmarcar la fenomenología de matriz husserliana en nuevos contextos (motivación ampliamente visible en la última década) y, por esa vía, de escudriñar su fecundidad, es un propósito enteramente legítimo y absolutamente inatacable. Pero este propósito no debe ser confundido con la oportunidad, que muchas veces se pone en total simetría con esta primera necesidad, de volver a la fenomenología productiva. A propósito de esta distinción entre fecundidad y productividad resuenan por todas partes las juiciosas palabras de Gaston Bache$\operatorname{lard}^{2}$, quien recordaba que la urgencia en deducir efectos de una determinada ciencia, en el sentido de volverla, de este modo, productiva, no hace más que denunciar nuestra obstinada permanencia en un dominio todavía distante del campo de la racionalidad científica o, para utilizar aquí el idiolecto de Husserl, en la esfera de la actitud pre-filosófica o natural. Si la condición para que la fenomenología se vuelva productiva es el abandono de sus decisiones teóricas de mayor alcance - pensamos especialmente en la renuncia a la actitud trascendental, que el propio Husserl parece admitir en el $\S 57$ de las Meditaciones cartesianas $^{3}$ - lo que surge de ese abandono es una ciencia positiva más entre otras, cuyos resultados no difieren sustancialmente de los indicadores de productividad ahí señalados. Partiendo de esta distinción operada en el plano conceptual, no decimos, sin embargo, que la fenomenología puede ser fecunda sin ser productiva o que esta última exigencia de productividad no se sitúe en el plano de la comprensión de la fenomenología como hacedora de la racionalidad científica. Lo que en todo caso no nos parece razonable es sostener que una tal reivindicada productividad se determine en base a una descaracterización absoluta de la naturaleza y función de las disciplinas implicadas o, incluso, en el cambio del que es el sentido más hondo de cada una de ellas. Efectivamente, y como consecuencia de esta descaracterización, vemos con cierto embarazo muchas veces a psicólogos que debaten, como fenomenólogos de parcos recursos, sobre cuestiones que están fuera de su alcance. Es lo que ocurre cuando se le exige a la psicología - la cual todavía opera en base a un mundo que se pone

\footnotetext{
${ }^{2}$ Vid. Gaston Bachelard, Épistémologie. Textes choisis, Paris, P.U.F.

${ }^{3}$ Vid. Edmund Husserl, Cartesianische Meditationen und pariser Vorträge, Hua I, Den Haag, Martinus Nijhoff, 1973.
} 
como supuesto no cuestionado- que se ponga como base necesaria para una teoría del conocimiento, para una filosofía trascendental. Del mismo modo, y con el mismo embarazo, asistimos al intento de situar la discusión fenomenológica en moldes que sólo pueden significar que ella sufrió una brusca mutación y que abandonó definitivamente el suelo propio de una filosofía trascendental para ponerse al modo de una disciplina positiva. En este capítulo en el que la fenomenología, para ser inmediatamente productiva, se presenta apenas como un régimen más de cientificidad entre otros, se inscriben los vanos intentos para pensar que la tarea esencial de la fenomenología se deja realizar integralmente en el contexto de una psicología fenomenológica, y se inscribe también la tesis de que la fenomenología trascendental no va a ser más que una psicología disfrazada ${ }^{4}$. Evidentemente, bastará una lectura más profunda del interés de Husserl por la psicología - y es de esto de lo que nos vamos a ocupar- para vencer inmediatamente estas interpretaciones y para instaurar definitivamente la idea de que para ser, en este singular contexto, productiva, la fenomenología no compite con la psicología, no reclama su espacio ni tampoco compara los respectivos resultados.

Sin embargo, sabemos bien la distancia que separaba a Husserl de una correcta comprensión psicologista de la Lógica. $Y$ también sabemos que la disciplina instaurada por Husserl se va a constituir como ciencia filosófica fundamental en la confrontación con la doctrina que determina la reducción de los principios de la ciencia a la naturaleza y a la constitución de nuestro espíritu. Pero incluso un neófito sabe que, sin embargo, la asociación entre fenomenología y psicología había sido solicitada por el propio Husserl, quien logró encontrar, en la psicología, una primera respuesta al problema del esclarecimiento filosófico de la lógica, que es donde se juega, prima facie, la cuestión de los fundamentos de la matemática y de la propia lógica - cuestión que constituyó, a su vez, una especie de antecámara que prefigura el objeto y anticipa el estilo de una disciplina fenomenológica madura y plenamente consolidada.

${ }^{4}$ Vid. Urbano Mestre Sidoncha, "É a Fenomenología uma Psicologia Disfarçada?", Phainomenon. Revista de Fenomenología 16/17 (2008). 
2. IMPORTANCIA DE LA PSICOLOGÍA DESCRIPTIVA Y SU SIGNIFICADO PARA LA TAREA DE REVISIÓN DE LOS FUNDAMENTOS DE LA PSICOLOGÍA: EL DEBATE ENTRE HUSSERL Y DILTHEY

En el semestre de verano de 1925, en el transcurso del célebre y fértil período de Friburgo, Husserl dicta un importante curso sobre psicología fenomenológica, incluido por Walter Biemel en el volumen IX de Husserliana. En ese curso, evidentemente Husserl no pretende contribuir con nuevos descubrimientos al inicio de una psicología fenomenológica que hasta esa fecha todavía no existía como régimen de cientificidad plenamente consolidado, pero sí pretende, por el contrario, hablar sobre los fundamentos conceptuales de la psicología a partir de una perspectiva fenomenológica. A pesar de esto, ha habido siempre coincidencia en considerar que a un tal enfoque le falta carácter sistemático y, por tanto, en que en todo caso se trataría de una orientación marginal en el seno de las orientaciones programáticas de la propia fenomenología. Sin embargo, falta decir que, más que comprometer la posibilidad o la necesidad de una tal perspectiva, lo que revela esa diagnosticada insuficiencia es más que nada la ausencia de una visión orgánica relativa a las cuestiones de la psicología que ahora se llama fenomenológica. La inexistencia de esa orientación sistemática sería, en el fondo $-\mathrm{y}$ si, en efecto, queremos atribuirle algún significado que supere la dimensión de lo puramente circunstancial-, la expresión fidedigna de esa ausencia más primitiva de un referente que, pensado retrospectivamente, prestara alguna unidad a las consideraciones de Husserl. Pero si, al margen de esto, la unidad de la discusión se agita como una condición que hace aparecer la perspectiva modal de la necesidad, incluso ahí no la podremos rechazar, teniendo en cuenta que se permite pensarla - toda ella- a partir de la unidad de la crítica que es dirigida hacia la presencia del naturalismo en la psicología. Éste es, ipso facto, el punto privilegiado a partir del cual la fenomenología, y sólo ella, asoma como disciplina que puede llamar a sí la sensible cuestión de la revisión de los fundamentos conceptuales de la psicología; y será éste, justamente, el desiderato esencial de Husserl en la prosecución de ese curso. Ésta es también, si quisiéramos, una primera aunque decisiva acepción para el proyecto de una psicología fenomenológica, que previene acerca de una perspectiva estrictamente científica que de ahora en adelante se vaya a arrojar sobre la psicología. 
Sin embargo, si en relación a esta asociación entre fenomenología y psicología - la cual, insistimos, debe ser puesta en primer lugar en la base de una inspección fenomenológica de los fundamentos de la propia psicología - todavía planea la sospecha de una - digamos- cierta insuficiencia (lo que nos permitiría tomarla simplemente como la expresión de una justa posición espuria y sin sentido), sea dicho, en forma de respuesta, que hay un conjunto de acontecimientos que ilustran bien la atmósfera intelectual de la época, mostrando, a la vez, que el entusiasmo inicial suscitado por la psicología formaba, más que una relación constituida en base a un acontecimiento aislado, una tendencia dominante que se extendió rápidamente en la época que anuncia el nacimiento de la propia fenomenología. Enumerémoslos de forma breve y sin preocupaciones de exhaustividad $^{5}$ : en 1860, un año después del nacimiento de Husserl, la psicología científica marca su aparición con los Elementos de psicofísica, de Fechner. En ese mismo año, con Broca, se señalan las primeras localizaciones cerebrales. En 1875, Franz Brentano publica la Psicología desde el punto de vista empírico, obra mayor que tendrá, como es bien sabido, profundo impacto sobre Husserl y sobre la propia definición del esbozo de la fenomenología en sus primeros años. El primer laboratorio de Psicología experimental, fundado por Wundt, data de 1879. En suma, fue esta situación, compleja en sus desarrollos internos pero ampliamente comprensible en sus rasgos de conjunto, la que encontró Husserl. La conjugación de estos acontecimientos determinará la plataforma de sentido a partir de la que Husserl realiza una crítica, científicamente relevante, al modelo naturalista de la psicología. Y esta crítica se revelará absolutamente necesaria, atendiendo al vínculo congénito que vincula la psicología al modelo y al método de las ciencias naturales ${ }^{6}$, en un momento que Husserl considera excesivamente marcado por las esperanzas que entonces se depositaban en la nueva psicología.

Esta crítica, que es la del propio Husserl, dialogará, permanentemente, con la perspectiva generada por parte de Wilhelm Dilthey en un célebre texto de 1894 titulado Ideen über eine beschreibende und zergliedernde Psychologie (Ideas acerca de una psicología descriptiva y analítica). No es que la inspección

\footnotetext{
${ }^{5}$ Para una profundización más completa de esos momentos, remito, entre la vastísima bibliografía relevante, al estudio de José Henrique Santos, Do Empirismo à Fenomenología. A crítica antipsicologista de Husserl e a ideia de lógica pura, Braga, Livraria Cruz, 1973.

${ }^{6} \mathrm{Cfr}$. Edmund Husserl, Phänomenologische Psychologie. Vorlesungen Sommersemester 1925, Hua IX, The Hague, Martinus Nijhoff, 1962, p. 4.
} 
fenomenológica que Husserl pretende llevar a cabo requiera el auxilio de la psicología descriptiva de Dilthey. Esta dependencia teórica y conceptual de la fenomenología en relación a una tesis positiva como es aquella que Dilthey desarrolla en el decisivo año de 1894, sería absolutamente intolerable para la coherencia interna de la perspectiva trazada por Husserl, la cual justamente se pretende libre de constreñimientos de ningún tipo. Lo que incita esta aproximación crítica a las tesis de Dilthey - a quien Husserl consideró, tal vez demasiado tarde, un hombre de intuiciones brillantes- es precisamente mostrar que sólo por la vía de la fenomenología puede ser efectivamente denunciada, en sentido pleno, y de una forma rigurosamente científica, esa tendencia naturalista de la psicología - tomada de las ciencias naturales-, y que otras denuncias que se le imputan, en su afán de consumar esta inspección, no hacen más que duplicar el error que pretendidamente se pretendía denunciar.

Muy sucintamente, y sin entrar en una discusión deliberadamente circunstanciada del pensamiento de Dilthey, lo que el autor le oponía a una psicología de confección naturalista - precisamente aquella que, según la lectura de Husserl, reivindica la misma importancia relativa y la misma dignidad institucional que entonces se le concedían a la física- es el hecho de que en ella se perdiese una cierta visión de conjunto de la vida mental, que sólo de modo artificial podría ser pensada atomísticamente al nivel de aconcimientos psíquicos aislados. Es éste el tejido de fondo teórico y motivacional que está en el origen, en Dilthey, de una inclinación hacia el terreno de una psicología descriptiva, como alternativa y como sustitución de su variante empírica, que utiliza, en la psicología, los métodos de las ciencias de la naturaleza. En efecto, la matriz teórica y conceptual que está en la base de la metodología de tales ciencias, cuando no lo está directamente vertida en el campo propio de la psicología, determina que la formulación de hipótesis sea puesta como elemento que conecta los datos sensibles de la experiencia externa y la conexión causal que, a su vez, no se nos da en el contexto de la propia experiencia. De entre las múltiples dificultades en que se deja enredar esta inscripción de la psicología en el espacio lógico de las Naturwissenschaften, la que más se granjeará la censura por parte de ese escepticismo radical gestionado por Dilthey (tal era el modo en el que Husserl veía el posicionamiento de Dilthey ante la psicología hipotético-causal ${ }^{7}$ ) es

\footnotetext{
${ }^{7}$ Cfr. Hua IX, p.14.
} 
la de incluso entender que esa estrategia inferencial, o ese pensamiento construido que trasciende el dato intuitivo, es una concepción que armoniza con las determinaciones específicas de la vida mental. "Hipótesis, por todas partes sólo hipótesis", se lamentaba Dilthey. Ahora bien, si es verdad que los méritos imputables a esa estrategia no merecen reparos cuando, por ejemplo, se dejan aplicar a la moderna física, no será menos cierto que ya no sucede lo mismo cuando esa estrategia se une, sin más, al espacio lógico de las ciencias del espíritu $^{8}$. Dice Husserl:

La psicología, y consecuentemente, todas las ciencias socioculturales, se refieren al nexo mental dado universalmente por la experiencia interna. La Innere Erfahrung no da una mera exterioridad, no conoce separación de partes que se pongan como elementos auto-suficientes. Sólo conoce estados internamente entrelazados, y entrelazados en la unidad de un nexo inclusivo, que es dado conjuntamente con los propios estados en cuanto nexo en la intuición interna. ${ }^{9}$

Es, pues, esta conexión viva, y no meramente artificial o construida, la que constantemente escapa a una psicología que importa sus procesos de la moderna física. Éste es el aspecto que inmediatamente convierte una unidad construida, a partir de fuera, en una unidad contrahecha, que a su vez se funda en la pura concatenación de procesos psíquicos aisladamente considerados. A esto mismo se refiere Dilthey:

En las ciencias de la naturaleza se nos ofrece un nexo natural sólo a través de deducciones suplementarias, mediante un complejo de hipótesis. Por el contrario, en las ciencias del espíritu, la base es la conexión de la vida anímica como algo originariamente dado [...]. En la experiencia interna son también dados los procesos de causación, de los lazos de las funciones, como miembros singulares de la vida psíquica, en un todo. Primordial es, aquí, la conexión vivida; secundaria la distinción de sus diversos miembros. Esto condiciona una diferencia muy grande de los métodos con que estudiamos la vida psíquica, la historia y la sociedad, en relación a los otros métodos por los que se obtiene el conocimiento de la naturaleza. ${ }^{10}$

Ahora bien, si es, pues, verdad que el dato aislado es para la física una simple abstracción -en la que hay un ejercicio perfectamente legítimo y absolutamente rutinario en el contexto de su propia orientación metodológica-, en la consideración de nuestra vida psíquica, por el contrario, esta abstracción hie-

\footnotetext{
${ }^{8}$ Devolvamos la palabra a Dilthey: "no âmbito psicológico, as hipóteses também não possuem a capacidade de realização de que deram provas no conhecimento científico-natural". Wilhelm Dilthey, Ideias acerca de uma Psicologia Descritiva e Analítica. Trad. Artur Morão: http://www.lusosofia.net/textos/dilthey_wilhelm_psicologia_descritiva_e_analitica.pdf, p. 16.

${ }^{9}$ Hua IX, p. 8.

${ }_{10}$ Wilhelm Dilthey, op. cit., p. 15.
} 
re de muerte una conexión íntima que, lejos de dejarse postular como hipótesis, antes que eso es originariamente dada en la experiencia interna como expresión de un significado inmanente. Según Husserl, los únicos que no podrían ser sensibles a las cuestiones planteadas por Dilthey ${ }^{11}$ son aquellos que todavía están bajo los efectos del hechizo de la orientación naturalista de la psicología dominante. El autor pretendía, por tanto, que las ciencias del espíritu tuvieran una metodología eminentemente comprensiva como alternativa a la reivindicada orientación explicativa de las ciencias de la naturaleza ${ }^{12}$. Como ahora comprendemos perfectamente, esto significaba el alejamiento definitivo de la psicología dominante y su sustitución por una psicología más concreta y menos abstracta o artificial, a la que el autor conseguirá llamar descriptiva. Dice Dilthey:

Sólo una ciencia que denominaré psicología descriptiva y analítica, en oposición a la explicativa o constructiva, puede librarnos de todas las dificultades expuestas. Entiendo por psicología descriptiva la exposición de los componentes y de los nexos que se presentan uniformemente en toda la vida psíquica humana desenvuelta, entrelazados en una única textura, que no es inferida o introducida por el pensamiento sino simplemente vivida. Esta psicología es, por tanto, la descripción y el análisis de una conexión que, de modo originario y siempre, se nos da como la propia vida. ${ }^{13}$

Puesta en esta transmutación que se opera en la propia intimidad de la concepción en psicología, encontrará Husserl el mérito fundamental que debe ser atribuido al abordaje de Dilthey:

La gran relevancia de la exposición de Dilthey -afirma Husserl en las lecciones de 1925- está, por encima de todo, en aquello que él dijo de positivo sobre la unidad de la vida psíquica como unidad de la experiencia viva y, consecuentemente, en la exigencia de una psicología descriptiva fundada netamente sobre la intuición. ${ }^{14}$

Este reconocimiento expreso de Husserl en relación al posicionamiento crítico de Dilthey, que está en la verdad en vez de constituir una anotación marginal, tomado inicialmente en cuanto a su pura forma deja adivinar uno amplío acuerdo en torno a la comprensión de la psicología; acuerdo que está edificado en el marco de las reservas de fondo que el primero tendrá que oponer a una psicología de tipo hipotético-constructiva. Pero eso no significa, evidentemente,

${ }^{11}$ Hua IX, p. 11.

12 "Os representantes da psicologia explicativa costumam apoiar-se nas ciências da natureza para justificar um tão amplo emprego de hipóteses. Mas já no início das nossas investigações queremos proclamar a pretensão das ciências do espírito de determinar autonomamente os seus métodos, de harmonia com o seu objecto." Wilhelm Dilthey, op. cit., p. 15.

13 Ibidem, p. 25.

${ }^{14}$ Hua IX, p. 10. 
que la disidencia que se iniciará más tarde entre los de los autores sea sólo la expresión de un lamentable equívoco. Es el propio Husserl quien inicialmente disocia esas reservas que tendrá que oponer a la imitación de la metodología diltheyniana - la cual considera no poder ser recomendada en ninguna circunstancia- de la justa admiración que se debe a su brillante intuición. En el breve espacio de este ensayo no es posible la tarea de trazar el mapa de las semejanzas y diferencias entre las concepciones de Husserl y de Dilthey, ni tampoco de la menos exigente misión de contrastar la justicia de las críticas que Husserl le dirige a Dilthey. De entre esas diferencias que se dejan determinar en un simple juego de confrontación de tesis positivas, aquí preferimos elegir la que se pone inmediatamente como la más emblemática de esa todavía insuficiente inspección de los fundamentos de la psicología, cuando ella es sobreentendida en el estricto contexto de una psicología descriptiva, y cuando una tal psicología descriptiva se deja incluso enredar en las paradojas que habían determinado la estruendosa ruina del psicologismo.

\section{INSUFICIENCIAS DEL TRATAMIENTO DILTHEYNIANO}

Incluso para una mirada todavía inicial, entender qué es la psicología descriptiva o cuál es la tarea que esencialmente le toca a ésta cumplir surge como la expresión de una diferencia insuperable entre los dos autores. Es cosa bien sabida que, para Husserl, una de las tareas de la psicología descriptiva es la de promover un distanciamiento en relación a una psicología todavía tomada en el sentido naturalista de ciencia de la experiencia, en un primero pero decisivo movimiento de radicalización que acompañará a la fenomenología y que la llevará, por último, a asumir su condición más perenne de filosofía trascendental. Este esfuerzo de radicalización se sustancializa, desde bien pronto, en una redefinición de su campo propio, que ya no es más el de los elementos reales de la vivencia sino más bien el de sus elementos ideales. Ahora bien, esta distinción elemental, aunque simultáneamente más fina entre elementos reales e intencionales de la vivencia, parece habérsele escapado a Dilthey, según la lectura muy juiciosa de Husserl.

Es incluso más grave, desde el punto de vista de sus implicaciones sistémicas, el diagnóstico de una correcta recaída de la psicología descriptiva de Dilthey en los absurdos que habían determinado la contundente crítica de Husserl a 
la psicología explicativo-causal. Una tal recaída tendría que ver, supuestamente, con la siguiente imposibilidad de fondo: si la psicología descriptiva es, ella misma, una ciencia positiva más entre las otras, ¿cómo justificar su pretensión de erigirse simultáneamente como fundamento de las ciencias socioculturales ${ }^{15}$, además de también erigirse como fundamento de la propia teoría del conocimiento ${ }^{16}$-teoría del conocimiento que en relación a las ciencias positivas se debe situar en un plano superior con el que todas guardan una misma distancia? Este contrasentido de partida es explicado por Husserl en los siguientes términos:

Que la teoría del conocimiento quiera volver inteligible, genéricamente y en principio, tanto la forma como la actividad cognitiva, en su interioridad psíquica, puede tener lugar en la producción de validez objetiva. Pero, ¿cómo puede ella alguna vez resolver este problema si depende de un procedimiento psicológico-empírico [es decir, si la hacemos depender de una psicología descriptiva que ve en la unidad del nexo psíquico el sustrato de todos los procesos cognitivos] que sólo le proporcionaría universalidades histórico-naturales, en vez de necesidades inviolables e intuitivamente evidentes? ${ }^{17}$

He ahí, pues, en su expresión más decisiva, las aporías en que se dejó enredar el propio psicologismo, y que parece conocer ahora una curiosa e inesperada reedición en los trabajos de Dilthey. Efectivamente, el autor de las Ideas acerca de una psicología descriptiva y analítica no acepta que la teoría del conocimiento se pueda formular en una total ausencia de contenido, sin tener por base el elemento psíquico. Mientras Husserl reconoce que, después de Kant, la teoría trascendental del conocimiento alcanzó una profunda influencia, habiéndose unificado en una perspectiva que rechazó categóricamente todo el ejercicio de fundación empírico-psicológica (aunque, por otra parte, no dejó de combatir como siendo una forma de ese criticado psicologismo ${ }^{18}$ ), Dilthey, a su vez,

15 "[...] A análise da realidade social e histórica, tão complexa, só poderá ser levada a cabo se esta realidade for, primeiro, desmembrada nos diversos sistemas de fins que a integram. [...] Mas a trama de tal sistema é apenas a conexão psíquica dos homens que nele cooperam. É portanto, em último termo, somente uma conexão psicológica." Wilhelm Dilthey, Ideen über eine beschreibende und zergliedernde Psychologie, Trad. portuguesa de Artur Morão: Ideias acerca de uma Psicologia Descritiva e Analítica: http://www.lusosofia.net/textos/dilthey_wilhelm_psicologia_descritiva_e_analitica.pdf, p. 30.

16 "Como o desenvolvimento de cada uma das ciências do espírito está ligado à constituição da psicologia, também não é possível conseguir a articulação das mesmas num todo sem compreender a urdidura psíquica em que se encontram entrosadas. Sem referência alguma à conexão psíquica em que se fundam as suas relações, as ciências do espírito são um agregado, um feixe disperso, e não um sistema [...] A mesma dificuldade pesa sobre a teoria do conhecimento [...] os factos espirituais que constituem o material da teoria do conhecimento não podem ser entrelaçados sem o pano de fundo de alguma representação do nexo psíquico." Ibidem, pp. 20s. En algunas páginas, puede leerse que "a conexão psíquica constitui o fundo do processo cognoscitivo e, portanto, este processo só nesta conexão psíquica pode ser estudado e determinado no seu alcance". Ibidem, p. 24.

${ }_{17}^{17}$ Hua IX, p. 19.

${ }^{18}$ Ibidem, p. 29. 
no acepta que la distinción entre materia y forma del conocimiento se pueda fundar autónomamente en relación a una realidad psíquica sino que, en las antípodas de esta tesis, sostiene que ella sólo encuentra su fundamento de determinación en la conexión anímica efectivamente dada en el campo de la experiencia interna.

Husserl se preguntaba: ¿Cómo puede una descripción, en la base de la mera experiencia interna $[. .$.$] , ser capaz de producir más que una simple com-$ prensión individual? [...] ¿cómo podrá alguna vez ir más allá de las vagas generalizaciones empíricas?" O, todavía en un otro y sugestivo paso: "en una psicología que depende meramente de la experiencia interna y de la descripción de la vida psíquica, ¿cómo llegamos a la universalidad de la ley ${ }^{19}$

Hay varios aspectos que merecen aquí una reflexión más penetrante:

i) esta inexactitud de las leyes psicológicas evidentemente no puede ser imputada, sin más, a una cierta, diríamos, inmadurez de la psicología, sobre todo en una circunstancia en que es llamada a estar presente entre otras ciencias positivas. Es, más bien, debido a una cuestión de principio por lo que se sitúa en un abandono del horizonte de sentido y de las posibilidades de realización de las ciencias experimentales ese desiderato de comprender todos los hechos a partir de un cierto número de leyes exactas. Insistimos: se trata únicamente de una imposibilidad de principio que nada tiene que ver con la idiosincrasia propia de la psicología. Como bien lo vio Theodor de Boer, "si las leyes de la lógica no fueran más que una rama particular de las leyes psicológicas, compartirían con éstas su carácter inexacto y provisional"20.

ii) Hay un segundo aspecto que, sin embargo, merece igualmente ser subrayado: lo que está puesto en causa en esta denuncia siempre reiterada de las consecuencias absurdas y escépticas del psicologismo no puede ser visto, sin más, como una ofensiva gratuita que sobreviene en un conflicto cada vez más hondo entre Husserl y la psicología. Lo que ahí se ve es nada más que la posibilidad de que se genere, vía método causal, el descubrimiento de leyes ideales. Por otra parte, la denuncia de este error sólo puede resultar beneficiosa para las ciencias; incluso, evidentemente y por la mayor parte de las razones, para

19 Ibidem, p. 11.

20 "If the laws of logic were nothing but a particular brand of psychological law, they would share in this inexact and provisional character." Theodore De Boer, The Development of Husserl's Thought, The Hage/Boston/London, Martinus Nijhoff, 1978, p. 218. 
las ciencias experimentales que, después de eximidas de esta inaceptable responsabilidad, dejan ahora restituirse su verdadero rostro. Digamos, siendo rigurosos, que ni en el auge de la fenomenología qua filosofía trascendental pretendió Husserl protagonizar esa descalificación de la psicología. A propósito del vínculo que ata la psicología a la fenomenología, y del beneficio que un tal vínculo representa para la consumación plena del esfuerzo de fundamentación de la propia psicología, dice Ricoeur que

la psicología no se comprende ella misma, [...] si no está anclada en la fenomenología. Es ésta quien la instruye sobre la esencia de la subjetividad; quien nada sabe sobre lo que es el yo puro -constituidor de toda la realidad [...] no sabrá lo que es el psiquismo, realidad constituida, generada en el seno del yo-puro. ${ }^{21}$

Algunas páginas más adelante, reaparece la misma tesis devuelta ahora a su expresión de máxima simplicidad:

La psicología - dice Ricoeur-, al igual que su objeto, es intra-mundana [...] ella no tiene sobre él ninguna inteligencia directa $[\ldots] .{ }^{22}$

iii) Se impone una última nota para la evaluación del contenido de sentido de esta experiencia interna que es permanente solicitada por la psicología descriptiva y analítica de Dilthey. Por su intervención, ésta no sólo corta de raíz, como ya hemos dicho, la posibilidad de alcanzar la universalidad de la ley, sino que también se identifica con un criterio todavía distante del tema de una conciencia intencional que, después de Brentano, constituirá claramente la cuestión central y el hilo conductor de la psicología ${ }^{23}$. Además de esto, esta experiencia interna, tomada de antemano en el sentido de una auto-interpretación vivida, todavía no se distancia, con total nitidez, de un mero método introspectivo, cuando una de las marcas más reveladoras del radical cambio de perspectiva operada por la entrada en escena de la fenomenología -incluso tomada en el sentido de una psicología descriptiva- es precisamente la propia fundación de

21 "[...] La psychologie ne se comprend pas elle-même, [...] si elle ne s'adosse pas à la phénoménologie. C'est elle qui l'instruit de l'essence de la subjectivité ; qui ne sait point ce qu'est le moi pur, constituant de toute réalité [...] ne saura pas ce qu'est le psychisme, réalité constituée, tissée dans I'environnement du moi pur". Paul Ricoeur, A L'École de la phénoménologie, Paris, J. Vrin, 1987, p. 43.

${ }^{22} \mathrm{He}$ aquí el fragmento completo : "La psychologie est [...] intra-mondaine comme son objet [...]; mais [...] elle n'a aucune intelligence directe de son objet ; elle ne se comprend pas elle-même, si elle ne comprend pas son objet comme une non-réalité réalisée." Ibidem, p.47. Cursivas mías.

${ }_{23}$ Son muchos los lugares en la vasta de Husserl en donde este reconocimiento y, en consonancia, esta herencia, son asumidos sin ambigüedades: Cfr., por ejemplo, Hua V, p. 59. 
esta distinción con una absoluta transparencia ${ }^{24}$. La objeción husserliana es, en este punto, incisiva: es cosa bien sabida que fue precisamente la imposibilidad de establecer con total claridad esta distinción entre experiencia interna y el mero ejercicio de introspección lo que determinó la célebre ofensiva protagonizada por Husserl contra el psicologismo.

Las descripciones de la fenomenología -escribe Husserl en los Prolegómenos a la Lógica pura- no se refieren a vivencias o clase de vivencias de personas empíricas; puesto que de personas, $[. .$.$] de vivencias mías o de otros, ella nada sabe ni conjetura$ cosa alguna; sobre tales vivencias, la fenomenología no levanta cuestiones, no busca determinaciones ni elabora hipótesis. ${ }^{25}$

\section{SUPERACIÓN DE LA PSICOLOGÍA DESCRIPTIVA}

A pesar de este registro eminentemente crítico - señal evidente de que el interés de Husserl por la psicología no desapareció después del primer embate crítico de 1900- está la circunstancia de haber considerado, un año después, que la tarea de la fenomenología podía ser cumplida en el espacio de un psicología descriptiva, y de que una tal psicología únicamente podía ser diferenciada de la fenomenología por parcas diferencias. Pero de esta constatación nada más que resulta que el problema se muestra ahora bajo un nuevo rostro: si la pulsión naturalista de la psicología puede ser investigada y denunciada por la fenomenología esto, finalmente, justifica la distancia de seguridad con la que Husserl siempre mantuvo la concepción de Dilthey; pero si, mientras tanto, la propia fenomenología - tomada en el sentido de una psicología descriptivaaún se ve contaminada (la metáfora es de Husserl en las lecciones de 1925) por ese reprobado naturalismo, esto significa que una tal inspección, para ser científicamente relevante, requiere ahora mucho más de lo que requiere una psicología; incluso si, por un lado, esta psicología se liberó de las flagrantes

\footnotetext{
${ }^{24}$ Esta sospecha en relación a una pretendida confusión entre reflexión fenomenológica y un simple método introspectivo, que informaría consecuentemente sobre la tesis de una supuesta superioridad del lenguaje fenomenológico, es enfáticamente subrayada por algunos autores. Tomemos el paso que se sigue como testimonio representativo de esta corriente de interpretación: "How does phenomenological reflection differ from introspection?", preguntará Bayne, para, pasadas apenas algunas páginas, reiterar esta sospecha en relación a las virtudes de la fenomenología husserliana: "[...] what reason do we have for thinking that the results of phenomenological reduction are more reliable than those of 'mere introspection'?". Tim Bayne, "Closing the Gap? Some Questions for Neurophenomenology", en Phenomenology and the Cognitive Sciences, Dordrecht, Kluwer Academic Publishers, 2004, pp. 3-7.

${ }^{25}$ Edmund Husserl, Hua XVIII, p. 13 / Investigações Lógicas. Primeiro Volume: Prolegómenos à Lógica Pura, Lisboa, Centro de Filosofia da Universidade de Lisboa, 2005, pp. 23s. Trad. Diogo Ferrer.
} 
insuficiencias de la versión empírica y genética, e incluso aunque, por otro lado, ella operara una especie de catarsis intelectual que la llevó a depurarse de las insuficiencias del abordaje diltheyniano. Pero esta fluctuación de sentido que acompaña la relación entre la entonces fenomenología naciente y la psicología más que ser el emblema para una especie de indecisión de fondo en cuanto al alcance y sentido de la disciplina fenomenológica es, de nuevo, el sentido de la propia marcha de la argumentación husserliana en su concreción plena. Efectivamente, en el momento en que se va paulatinamente rediseñando el mapa de la todavía emergente escuela fenomenológica, tal comprensión se determina precisamente en el contenido de sentido que se imputa a la propia psicología, la cual ahora se deja tomar ya no en el sentido naturalista de ciencia de la experiencia -es decir, en una variante genética llena todavía de presupuestos naturalistas- sino en su valor descriptivo o puro, que lanzará la disciplina de Husserl hacia una creciente exigencia de radicalidad que es el punto en donde, paradójicamente, se determinará finalmente un alejamiento igualmente progresivo en relación a los terrenos aún mundanos de la psicología. En efecto, Husserl fue paulatinamente ganando conciencia de que estaba equivocado al caracterizar, en la base y en los términos de una auto-objetivación psicológica, lo que eran las esencias de los actos cognitivos y de los respectivos objetos correlativos. Se dio cuenta, por tanto, de que la fenomenología pura o trascendental debe esencialmente sustituir a la psicología descriptiva, a pesar del evidente beneficio que una tal concepción ya representa en relación al estadio primitivo de la psicología genética, y aún a pesar de la importancia del interés ahí declarado en interrogar la subjetividad de la consciencia. Pero este último alejamiento, que en sí mismo incluso es una respuesta más eficaz al llamamiento en pro de una radicalidad cada vez más profunda, no nos debe hacer olvidar que esta psicología ya era, por sí sola, revolucionaria cuando era inicialmente comparada con la psicología corriente de aquel tiempo, y que en las Logische Untersuchungen encontramos extensas aclaraciones sobre la esencia de la intencionalidad que eran desconocidas antes de Husser ${ }^{26}$.

${ }^{26}$ A este propósito, Theodore de Boer recuerda que "we must not forget that this psychology by itself was already revolutionary when compared to the psychology current at that time: in Logische Untersuchungen we find many insights into the essence of intentionality that were unknown before Husserl". Theodore De Boer, op. cit., pp. 434s. 
A pesar de este alejamiento progresivo en relación al terreno de la apercepción psicológica, será en la segunda edición de las Investigaciones lógicas de 1913 - por consiguiente, edición contemporánea al primer volumen de Ideen- en donde, finalmente, explicará la ausencia, del término "descriptivo", no pretendiendo ahí más que prevenir acerca de un inoportuno y peligroso regreso al psicologismo y acerca de la consiguiente depreciación de la fenomenología a la vejatoria condición de ciencia particular con un alcance parcial. Uno de los puntos que permite determinar, con absoluta nitidez, la importancia singular de esa revisión del texto de las Logische Untersuchungen para la plena consumación del proceso de maduración interna de la propia fenomenología, finalmente comprendida como filosofía trascendental, está en la separación del propio concepto de conciencia de todas sus determinaciones empíricas -marca irrecusable de una fenomenología que aún no logró el propósito de distanciarse de los resultados continuamente unilaterales de la psicología ${ }^{27}$. Para parafrasear a Eduard Marbach, Husserl lleva a cabo la determinación del concepto de conciencia en dependencia con la del concepto de yo empírico, lo que significa que la conciencia es tomada en las Investigaciones lógicas por referencia al yo empírico o, dicho de otro modo, en apercepción empírica ${ }^{28}$. En el Prefacio de la segunda edición de Logische Untersuchungen, y refiriéndose explícitamente a la comprensión ahí expuesta con respecto a una fenomenología todavía entendida como psicología descriptiva, Husserl es lento en la identificación de las dificultades ante las que la psicología zozobró. El texto, que es largo en su articulado pero escueto en su objetivo, reza así:

Me percaté de sus deficiencias después de la publicación, y, de hecho, pronto encontré la ocasión para plantear objeciones a mi propia designación de fenomenología como

\footnotetext{
27 "Le nouveau texte [de 1913] précise [...] que la phénoménologie étudie des faits vécus de conscience (Erlebnisse) 'dans leur pure généralité' et non come des faits réellement éprouvés, saisis empiriquement par des êtres conscients, au sein d'une nature". Gaston Berger, "Le Cogito dans la Philosophie de Husserl", Paris, Montaigne, p. 31. Cursivas mías.

${ }^{28}$ Vid. Eduard Marbach, Das Problem des Ich in der Phänomenologie Husserls, Den Haag, Martinus Nijhoff, 1974. No obstante, esta continuidad no debe oscurecer, en definitiva, el papel fundador de las Logische Untersuchungen y de su contribución a la constitución de la propia fenomenología: "Même dans la première édition, et malgré l'imprécision qui est inévitable au début d'une recherche nouvelle, on voit bien que la phénoménologie est tout autre chose qu'une forme particulière de psychologie". Gaston Berger, op. cit., p. 31. En otro pasaje, la importancia del "trabajo preparatorio" de las Logische Untersuchungen está todavía más reivindicada: "Les premières corrélations reconnues dans les Recherches logiques sont encore 'dans le monde', mais elles ouvrent la voie à la découverte de la corrélation fondamentale, celle qui rattache l'ensemble du monde au sujet transcendental qui porte le monde 'à titre d'unité de sens'". Idem.
} 
psicología descriptiva ${ }^{29}[\ldots]$ La descripción psicológica, llevada a cabo en la experiencia interior [en la inmanencia real, es decir, en la inmanencia en la conciencia del hombre y en el fenómeno psíquico real] aparece puesta al mismo nivel que la descripción, llevada a cabo en la experiencia exterior, de los procesos naturales exteriores; la descripción psicológica es, por otro lado, puesta en oposición a la descripción fenomenológica, de la que quedan enteramente excluidas todas las interpretaciones trascendentes de los datos inmanentes, e incluso aquellas interpretaciones que los toman como "actividades y estados psíquicos" de un yo real. ${ }^{30}$

En efecto, la necesidad de radicalidad en fenomenología - necesidad que en sí misma es incluso un objetivo intermedio alcanzado por medio de la reducción trascendental- apunta a una esfera de otra imanencia en relación a la cual está envuelta en una comprensión todavía psíquica de esa misma esfera de inmanencia. Husserl lo dice categóricamente en el párrafo 14 de las Cartesianische Meditationen. El texto reza así:

Tal vez se dirá que seguir esta dirección de investigación corresponde a hacer simplemente descripción psicológica, fundada sobre la experiencia puramente interna de mi propia vida consciente [...]. Pero esta psicología puramente descriptiva [...] no es, ella misma, fenomenología trascendental, en el sentido en que la definimos por la reducción trascendental. ${ }^{31}$

Esto significa, pues, que combatir el naturalismo encontrará en la "simple" oposición a la "naturalización" de las ideas un importante momento para su concreción. Pero esto no basta: es necesario que esa oposición, ya vertida en los Prolegomena, se haga acompañar ahora por una fuerte reacción contra la propia naturalización de la conciencia ${ }^{32}$.

${ }^{29}$ A propósito de esta designación de la fenomenología como psicología descriptiva, Theodore De Boer hace notar, no sin algún sentido de humor, que "it is not likely that Husserl wrote many sentences he regretted more than this one". Theodore De Boer, op. cit., p. 207.

${ }_{30}$ Edmund Husserl, Hua XVIII, pp. 12s / Investigações Lógicas. Primeiro Volume: Prolegómenos à Lógica Pura, p. 23.

${ }^{31}$ Cfr. Edmund Husserl, Hua I.

32 "The (natural scientific) "thing in itself" was presupposed behind the intentional object; beneath the phenomenological sphere was the physical body, and via this body the rest of the physical world. [...] Consciousness, which is isolated from the rest of reality trough the epoché, remains as part of that reality. [...] Therefore, the struggle against naturalism was doomed to sterility and did not represent a radical critique." Theodore De Boer, op. cit., p.502. En otro pasaje, puede leerse "Between 1900 and 1913, Husserl would have realized that [...] he would have to overcome naturalism or positivism in a more radical way: he would have had to argue that consciousness [...] is not part of the causal natural world. And this is a central thesis of transcendental idealism." Herman Philipse, "Transcendental Idealism", en Barry Smith / David Woodruff Smith (eds.), The Cambridge Companion to Husserl, Cambridge, Cambridge University Press, 1995, p. 245. 


\section{PSICOLOGÍA FENOMENOLÓGICA Y FENOMENOLOGÍA TRASCENDENTAL}

Las mismas razones explicarán el cambio de actitud -una Einstellungsänderung, como dice Husserl- en relación al terreno positivo, aunque eminentemente reflexivo, de la disciplina que se pone como sustrato a partir del cual puede ser establecida una psicología empírica rigurosamente científica, y que ocupa la atención de Husserl de forma más sistemática entre los años de 192528. Evidentemente me refiero al tema de la constitución de una psicología pura o psicología fenomenológica, delimitada temáticamente por el instrumento de la reducción fenomenológica y dirigida al campo de las puras experiencias psíquicas. La necesidad de un tal proyecto que, como Husserl hizo notar, no brotó internamente de las necesidades de la propia psicología -es decir, que no fue fundada a partir del deseo de constituir una psicología radicalmente científica-, comenzó a ganar forma, históricamente, en el trabajo pionero de Locke, poniendo el acento tónico de la investigación en aquella experiencia que se da en el espacio de una autoreflexión del sujeto sobre sí mismo en el método cartesiano de la duda que, según Husserl, constituyó la primera y más decisiva etapa en el sentido del desvelamiento de una subjetividad trascendental y también en el del descubrimiento fundamental de Brentano, materializada en la reapreciación del concepto escolástico de una intencionalidad tomada, de ahora en adelante, como característica esencial de los fenómenos mentales. Una tal psicología fenomenológica, necesaria para la prosecución plena de la tarea de revisión de la psicología empírica, se ocupará de esos mismos temas que la fenomenología trascendental llamará finalmente a sí y sobre los que lanzará la misma perspectiva esencial, eidética, volviendo aparentemente obsoleta una putativa recomendación de que tales disciplinas deberían seguir un desarrollo sistemático propio y, finalmente, justificando la cuestión que consiste en saber si la tarea de la filosofía trascendental no puede ser integralmente realizada en el espacio de una tal psicología pura. No obstante, y pese a esa cercanía con respecto al terreno autónomo de la fenomenología trascendental, la psicología todavía se pone a sí misma como ciencia positiva que opera en el terreno de la actitud natural y, por tanto, asoma como una perspectiva que todavía se deja enredar en el círculo epistemológico o trascendental. Incluso tomada en el sentido de una disciplina eidético-fenomenológica, la psicología todavía ostentará el rasgo distintivo de todas las ciencias positivas, que es precisamente el de 
operar en una impropia ingenuidad trascendental o, para utilizar la nomenclatura de Husserl en otro registro pero igualmente sugestivo, en una manifiesta ausencia de radicalidad, que la colocará en un plano de exigencia muy inferior a aquel que debe ser requerido para la constitución de la base de la filosofía trascendental. Es ésta, pues, la ocasión para devolver la palabra a Husserl.

En el tercer volumen de Ideen, que tiene como subtítulo Die Phänomenologie und die Fundamente diere Wissenschaften (La fenómeno-logía y los fundamentos de las ciencias), el autor considera

que es de una necesidad fundamental, y de una importancia cardinal para la filosofía, que ascendamos al reconocimiento de que es necesario distinguir la eidética de los estados de conciencia, que es una pieza de la ontología racional de la psique, de la eidética de una conciencia trascendentalmente purificada $[\ldots] .^{33}$

La psicología, afirma decisivamente Husserl, no puede ser puesta como fundamento de la filosofía trascendental. En otras palabras: la subjetividad hallada en el espacio de esta psicología fenomenológica todavía no está en la posición de llamar para sí, radicalmente y de forma enteramente consecuente, la cuestión epistemológico-trascendental de los fundamentos de todo el conocimiento mundano, incluyendo el suyo propio $^{34}$. Se trata, de este modo, de una ciencia que últimamente no se justifica ni se comprende a sí misma, y que vuelve urgente la cuestión de una nueva reducción, una reducción trascendental o reducción fenomenológica enteramente universal, que sea efectivamente dirigida a todo el mundo de la experiencia y que se refiera a toda la cognición positiva y a las ciencias que se colocan directamente en su estera. Únicamente por medio de este método enteramente radical - método que inaugura una experiencia de un tipo absolutamente nuevo-, puede la fenomenología trascendental cumplir el destino que corresponde a toda la filosofía científica y evitar los equívocos de la psicología, en sus variantes empírica y eidética y, con eso, desterrar definitivamente las contradicciones del círculo epistemológico y de toda suerte de naturalismo.

Con todo, esa diagnosticada insuficiencia no determina su caducidad y su absoluto descrédito en cuanto régimen de cientificidad. Aunque incrustada en el

${ }^{33}$ Hua V, p.75.

34 "The subjectivity of phenomenological psychology is [...] not yet in a position to address the transcendental-epistemological question of the foundation of all worldly knowledge, including its own." Steven Galt Crowell, Husserl, Heidegger and the Space of Meaning. Paths toward Transcendental Phenomenology, Northwestern University Press, Evanston/Illinois, 2001, p. 172. 
espacio lógico de las ciencias positivas, Husserl consideraba que a la psicología fenomenológica no debería serle rechazada su incuestionable utilidad propedéutica como preparación y modo de acceso indirecto para una fenomenología filosófica, la cual es para la psicología empírica como una geometría a priori lo es para la física empírica. Esto significa que, de la misma manera que fueron razones extrínsecas a la propia fenomenología las que habían preparado la llegada de una psicología pura, de nuevo son razones exteriores al proyecto de una tal psicología - dicha- fenomenológica las que habían determinado el cambio de actitud a la que acabamos de referirnos.

Por todo ello, no es extraño que Husserl haya recordado continuamente que la nueva perspectiva inaugurada por la fenomenología difícilmente podría ser convocada a partir de la designación antigua de psicología (la palabra histórica "psicología" dejó de ser adecuada, recuerda Husserl en el curso de $1925^{35}$ ), so pena de ser invocada una cierta perspectiva cuya superación al final constituyó la causa más cercana para la propia fundación de la fenomenología en su configuración madura.

\section{CONSIDERACIONES FINALES: LA IMPORTANCIA DE LA FILOSOFÍA FENOMENOLÓGICA PARA LA PSICOLOGÍA}

La interna clarificación o la consolidación del terreno de la psicología, que implica la denuncia sistemática y reiterada de su bien conocida y congénita pulsión naturalista, y que se concretiza mediante una inspección absolutamente rigurosa de sus fundamentos, impone un punto de vista que ya no se deja confinar al espacio propio de esa misma psicología. La contienda entre Husserl y Dilthey, al par de las diagnosticadas insuficiencias -diría-mos- epistemológicas, todavía asociadas al proyecto de una psicología fenomenológica, muestra que la distancia que antes apartaba a una psicología empírica y genética de una psicología descriptiva y comprensiva puede ahora ser desarrollada, mutatis mutandis, en la relación entre una psicología eidética y la propia fenomenología trascendental. Diríase, pues, en este momento que, más que cumplir el desiderato fundamental de la clarificación del campo autónomo de la investigación

\footnotetext{
${ }^{35}$ Hua IX, p. 32.
} 
psicológica, lo que hace una fenomenología que ostenta aún las marcas de lo psicológico, de lo mundano y de los presupuestos naturalistas que siempre lo acompañan, es enfeudarse en un horizonte de sentido que todavía no permite acomodar, o por lo menos no de forma decisiva, las preocupaciones relativas a las fundaciones conceptuales de la psicología. La crítica al naturalismo en la psicología -crí-tica que se tiene como orientación que es común a Dilthey y a Husserl y que irrumpe por vez primera en el espacio lógico de una comprensión más penetrante del contenido de sentido de la propia psicología- es, de modo indiscutible, la rampa de lanzamiento que sistemáticamente había preparado el camino para una inspección científicamente relevante al reprobado modelo naturalista de esa ciencia y, de este modo, para una tal reinterpretación o reordenación de fondo del campo autónomo de la investigación psicológica, capaz de proyectarlo en un plano de sentido enteramente nuevo y totalmente vaciado de manifestaciones unilaterales. Lo que de aquí resulta, por tanto, es que sólo por la vía de la desactivación plena de la actitud natural y de la desactivación de la filiación de la psicología empírica - consen-tida e instigada de forma abierta y declarada o meramente solicitada de modo aún velado, como sucede por parte de la psicología fenomenológica-, sólo por esta vía de la desactivación plena de esa filiación de la conciencia con una región mundana puede rendirse, a título definitivo, el indispensable soporte teórico para una psicología que entonces, y sólo entonces, se verá definitivamente liberada de atavismos y de las múltiples incomprensiones de fondo en que ella sucesivamente se deja, por culpa propia, enredar. 